\begin{tabular}{|c|c|}
\hline Title & Efficient Laguerre Gaussian mode conversion for orbital angular momentum resolved spectroscopy \\
\hline Author(s) & Toda, Y .; Shigematsu, K.; Y amane, K.; Morita, R. \\
\hline Citation & $\begin{array}{l}\text { Optics communications, 308, 147-151 } \\
\text { https://doi.org/10.1016/.optcom.2013.05.050 }\end{array}$ \\
\hline Issue Date & 2013-11-01 \\
\hline Doc URL & http:/hdl.handle.net/2115/54705 \\
\hline Type & article (author version) \\
\hline File Information & Optics_Communications_yt.pdf \\
\hline
\end{tabular}

Instructions for use 


\title{
Efficient Laguerre-Gaussian mode conversion for orbital angular momentum resolved spectroscopy
}

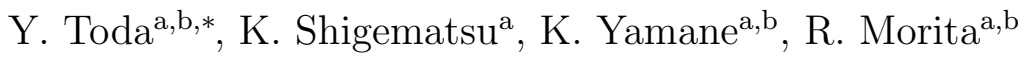 \\ ${ }^{a}$ Department of Applied Physics, Hokkaido University, N13W8 Kita-ku, Sapporo, Japan \\ ${ }^{b}$ Japan Science and Technology Agency, CREST, 5, Sanbancho, Chiyoda-ku, Tokyo, \\ 102-0075, Japan
}

\begin{abstract}
The orbital angular momentum (OAM) resolved spectroscopy is being applied to various research fields associated with optical vortices or LaguerreGaussian (LG) beams, in which the key technique is to convert LG beams to a fundamental Gaussian $\left(\mathrm{HG} \equiv \mathrm{HG}_{00}\right)$ beam. In this work, we propose a method to realize efficient mode conversion by using spiral phase modulations. Numerical simulations and experiments are carried out to compare our proposal with the standard technique using rotational phase modulations. The results reveal that our proposed method improves the uniformity of the conversion efficiency across various OAM, allowing us to obtain precise OAM spectra.
\end{abstract}

Keywords:

PACS: 42.25.Fx Diffraction and scattering, 42.50.Tx Optical angular momentum and its quantum aspects, 42.62.Fi Laser spectroscopy

\footnotetext{
*telephone/fax:+81-11-706-6627

Email address: toda@eng.hokudai.ac.jp (Y. Toda)
} 


\section{Introduction}

Helical beams whose cross sectional phase is described by $\exp (i \ell \phi)$, where $\phi$ is the azimuthal angle and $\ell$ takes any integer value, can be specified by the photon state with orbital angular momentum (OAM) $\ell \hbar$ or by the topological charge $\ell$ of dislocation for vortex fields [1]. The former has extended the potential of laser applications $[2,3,4]$ while the latter has linked the optics field with various other fields of science including topology in mathematics $[5,6]$. The single-ring helical beams are also associated with the Laguerre-Gaussian (LG) modes $\mathrm{LG}_{\ell}$ of the paraxial wave equation, where the topological charge $\ell$ denotes the azimuthal mode index of the LG and connects to the OAM.

LG beams are commonly generated by applying spatial phase modulations to a Hermite-Gaussian $(\mathrm{HG})$ beam $\left(\mathrm{HG}_{00} \equiv \mathrm{LG}_{0}\right)$ using a diffraction optical element such as a phase plate or a spatial light modulator (SLM) $[7,8,9]$. On the other hand, $\ell$ of the LG beam can be evaluated by OAMresolved ( $\ell$-resolved) spectroscopy, in which the analyzed beam is decomposed into a series of $\mathrm{LG}_{\ell}$ modes with corresponding intensities [10]. The technique measures the intensity of the $\mathrm{HG}$ component that is obtained by the $\mathrm{LG}_{\ell}$ to $\mathrm{HG}\left(\mathrm{LG}_{\ell} \rightarrow \mathrm{HG}\right)$ mode conversion using an approach that is the reverse of that used for generating an LG beam. Since the phase singularity of LG beams generates a ring-shaped intensity profile, only the HG component can be selected after the beams have passed through a spatial filter or singlemode fiber. The converted fundamental HG beam is also useful in terms of adopting the technique for various applications, such as telecommunication, optical storage, and laser spectroscopy $[11,12,13,14,15]$. Moreover, recent progress on optical geometric transformation using an SLM has made it pos- 
sible to obtain a spatially separated OAM spectrum that shows the relative intensities of $\mathrm{LG}_{\ell}$ simultaneously [16].

Although these techniques allow us to evaluate the OAM distribution of the analyzed beam, the precision of the spectrum depends strongly on the conversion efficiency and its uniformity for various OAM. For example, the spatial filtering of the HG component is based on the difference between the spatial intensity profiles of HG and LG beams. Therefore, the imperfect components of the converted HG reduce the accuracy of the spectrum. Furthermore, owing to the diffractive optical element in the techniques, the degree of conversion usually shows a strong OAM dependence with a fixed propagation distance, leading to variation of the conversion efficiency.

In this paper, we investigate the $\mathrm{LG}_{\ell}$ to $\mathrm{HG}$ mode conversion process using an SLM for precise OAM spectroscopy. In order to obtain precise OAM spectra, we propose a mode conversion by using spiral phase modulations. With conventional rotational phase modulation, an LG beam with a higher OAM shows the imperfection of the conversion. The deviation from the ideal HG is noticeable as the OAM of the analyzed LG beam is increased. By utilizing spiral phase modulation, near-complete conversion to an ideal HG is successfully realized, even in an LG with a higher OAM. The uniformity of the conversion efficiency across various OAM is accounted for by optimizing the degree of spiral phase.

\section{Theoretical}

In this work, we consider the OAM spectra obtained with $\mathrm{LG}_{\ell}$ to $\mathrm{HG}$ mode conversion using an SLM. In this section, we perform model calcula- 
tions of $\mathrm{LG}_{\ell} \rightarrow \mathrm{HG}$ to understand where the imperfect mode conversion arises using rotational phase modulations and how its magnitude depends on the OAM. We also compare the results with those using spiral phase modulations. Since $\mathrm{LG}_{\ell} \rightarrow \mathrm{HG}$ can be considered a diffraction phenomenon, we analyze the conversion process using the wave optical approach described below.

We first introduce the basic equation for the LG modes in cylindrical coordinates. Within the framework of a paraxial wave approximation, the amplitude of a collimated $\mathrm{LG}_{\ell}$ beam with a beam radius $w$ can be expressed by $[1]$

$$
u_{\ell}(r, \varphi)=\sqrt{\frac{2}{\pi|\ell| !}} \frac{1}{w}\left(\frac{r}{w}\right)^{|\ell|} \exp \left(-r^{2} / w^{2}\right) \exp (i \ell \varphi),
$$

where we neglect the wavefront curvature and Gouy phase. The corresponding intensity shows a single-ring beam profile, in which the center of the ring is associated with the phase dislocation of the optical vortex with topological charge $\ell$. The radius of the ring, which is defined by the distance of the peak intensity from the center, is given by

$$
\rho=\sqrt{\frac{\ell}{2}} w
$$

indicating that the ring radius increases with $\ell$.

For $\mathrm{LG}_{\ell} \rightarrow \mathrm{HG}$, the LG beam is diffracted by a computer generated hologram (CGH) on the SLM. In the experiment, a grating-based CGH is employed to spatially isolate the diffraction beam. For simplicity, in the calculation, only the rotationally symmetric part of the modulation is taken into account as $\phi(r, \varphi)$. Note that the absence of the grating does not affect the intensity profile of the diffracted beam. The diffraction integral of the input 


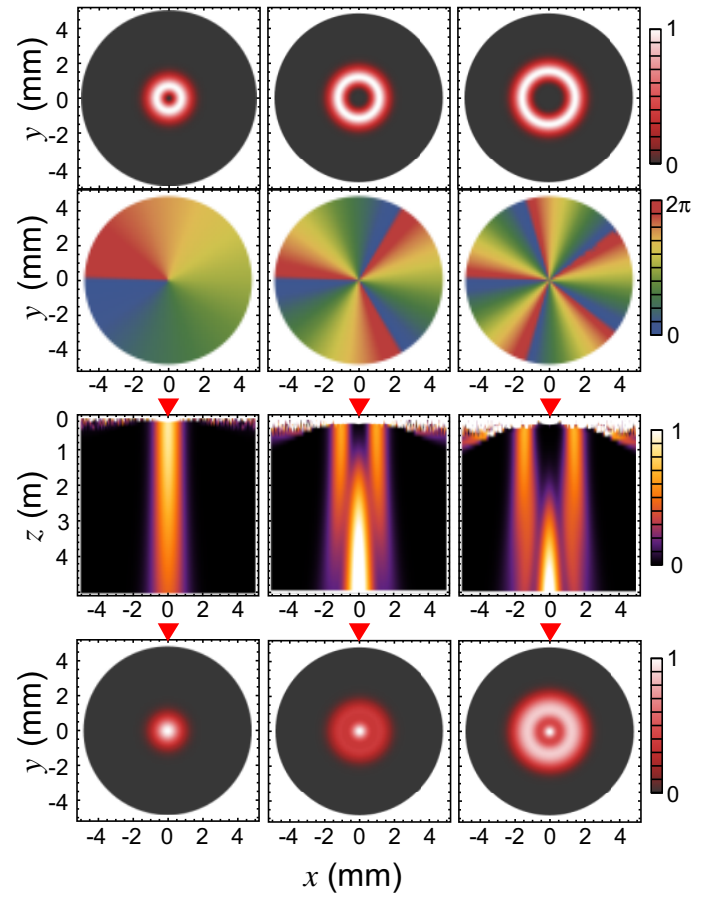

Figure 1: Model calculations of $\mathrm{LG}_{\ell} \rightarrow \mathrm{HG}$ mode conversion for input LG beams with $\ell_{i}=1$ (left), 3 (middle), 5 (right). Incident LG beams (top, $x y$-plane) at $z=0$ are converted into HG beams (bottom, $x y$-plane) by rotational phase modulations with $\ell_{c}=1$ (left), 3 (middle), 5 (right) (2nd row, $x y$-plane). The 3rd row shows the intensity profiles ( $x z$ plane) of the converted beams propagating along $z$. The bottom shows the cross-sectional views of the diffracted HG beams at $z=3000 \mathrm{~mm}$.

LG amplitude $u_{\ell_{i}}\left(r_{1}, \varphi_{1}\right)$ at the position $\left(r_{2}, \varphi_{2}, z\right)$ is given by

$$
\begin{gathered}
u\left(r_{2}, \varphi_{2}, z\right)=\frac{i}{\lambda} \int_{C G H} u_{\ell_{i}}\left(r_{1}, \varphi_{1}\right) \phi\left(r_{1}, \varphi_{1}\right) \frac{\exp (-i k L)}{L} K(\theta) d S \\
L=\sqrt{\left(r_{2} \cos \varphi_{2}-r_{1} \cos \varphi_{1}\right)^{2}+\left(r_{2} \sin \varphi_{2}-r_{1} \sin \varphi_{1}\right)^{2}+z^{2}}
\end{gathered}
$$

where $\lambda$ is the wavelength, $k$ is the wavenumber, and $K(\theta)$ is the inclination factor, which can be approximated by $z$ in a paraxial wave approximation.

Figure 1 shows the results of numerical calculations using rotational phase 
modulation $\phi(r, \varphi)=\exp \left(-i \ell_{c} \varphi\right)$ for the ideal single-mode LG beams with $\ell_{i}=1$ (left), 3 (middle), and 5 (right), where the light source is assumed to be a He-Ne laser $(\lambda=633 \mathrm{~nm}, w=1 \mathrm{~mm})$. The top panels show the crosssectional intensity profiles of the input LG beams. For $\mathrm{LG}_{\ell} \rightarrow \mathrm{HG}$, the OAM of a CGH $\left(\ell_{c}\right)$ is selected to cancel $\ell_{i}$ (i.e. $\ell_{c}=\ell_{i}$ ) as shown in the 2nd-row. As a result, the diffracted beam propagates as an HG-phased beam whose initial intensity is given by a ring associated with the input LG. The 3rd row of the figure shows the propagation dynamics of the diffracted beam, in which the split in the beam around $z=0$ indicates the initial ring-shaped HG. As the beam propagates along the $z$-axis, a single peak emerges at the center $(x=0)$, which is responsible for a complete $\mathrm{LG}_{\ell} \rightarrow \mathrm{HG}$. Here we emphasize that peak shifts appear along $z$ with increasing $\ell_{i}$. In the diffraction process, the intensity conversion is achieved only when the ring beam overlaps its amplitude at the center. Since the ring radius increases with $\ell$ as is clear from Eq. (2), a beam with a higher $\ell_{i}$ should propagate over a greater distance to complete the conversion. The shift in the appearance of the peak thus indicates that the $\mathrm{LG}_{\ell} \rightarrow \mathrm{HG}$ for an $\mathrm{LG}$ with a higher $\ell_{i}$ is incomplete within a finite propagation distance. The incomplete conversion can also be confirmed in the cross-sectional intensity profiles of the diffracted beams at $z=3000 \mathrm{~mm}$ (bottom of Fig. 1), in which the ring-like structure around the peak corresponds to the incomplete component of the mode conversion, which becomes significant with increasing $\ell_{i}$. The result indicates that the integrated peak intensity is reduced to less than $10 \%$ of the total intensity for $\ell_{i}=5$. The ring component thus reduces the selectivity of the spatial filtering of the OAM spectroscopy, and its OAM dependence increases the deviation 


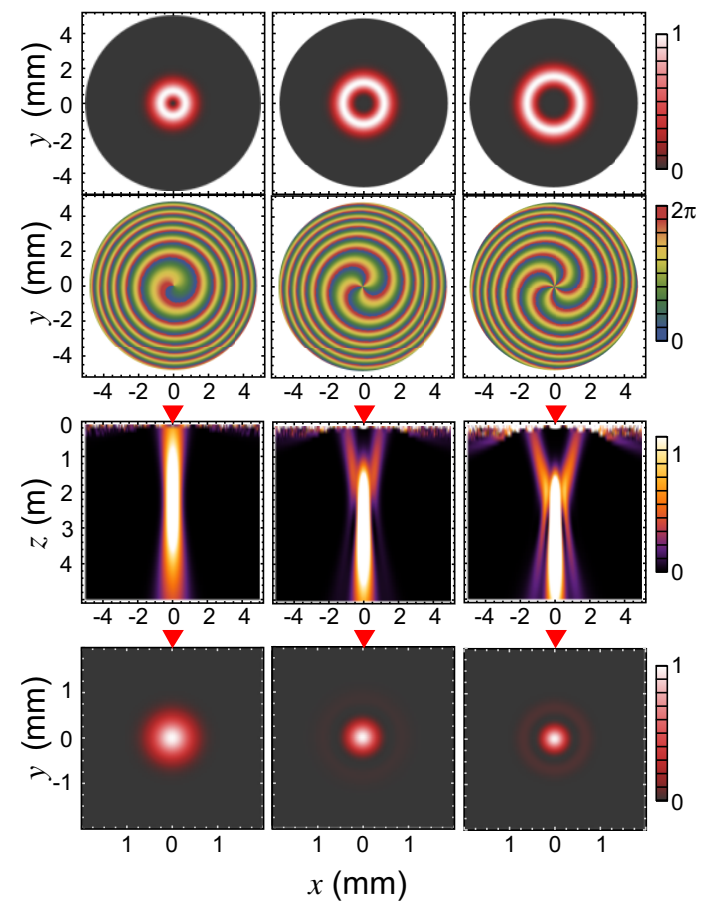

Figure 2: The same as Fig. 1 but using spiral phase modulations for $\ell_{c}=1$ (left), 3 (middle), 5 (right) with $f=3000 \mathrm{~mm}$, instead of rotational phase modulations.

of the spectral shape. Although perfect conversion should be realized within the infinite limit of the propagation distance, an actual experiment must complete the conversion process within a finite and shorter distance.

The efficient overlapping of a ring beam within a finite length is achieved by using a focusing lens. Alternatively, we can employ an spiral phase modulation that allows both focusing and $\mathrm{LG}_{\ell} \rightarrow \mathrm{HG}$ mode conversion simultaneously in simpler optical setup. Moreover, by taking advantage of the flexibility of the SLM, the focusing length can be controlled to compensate the conversion efficiency for various OAM. Figure 2 shows the results of the same numerical calculations as in Fig. 1 but, instead of rotational phase modu- 
lation, using spiral phase modulation $\phi(r, \varphi)=\exp \left(-i \ell_{c} \varphi\right) \exp \left(-i \frac{k r}{2 f}\right)$, where the focusing length $f=3000 \mathrm{~mm}$ was used. The cross-sectional phase distributions of the spiral phase modulation are shown in the 2nd row. The 3rd and bottom rows, respectively, show the propagation dynamics of the diffracted beams and their cross-sectional intensity profiles at $z=3000 \mathrm{~mm}$. The $\mathrm{LG}_{\ell} \rightarrow \mathrm{HG}$ mode conversion is nearly completed within $z=3000 \mathrm{~mm}$ even in the higher $\ell_{i}$.

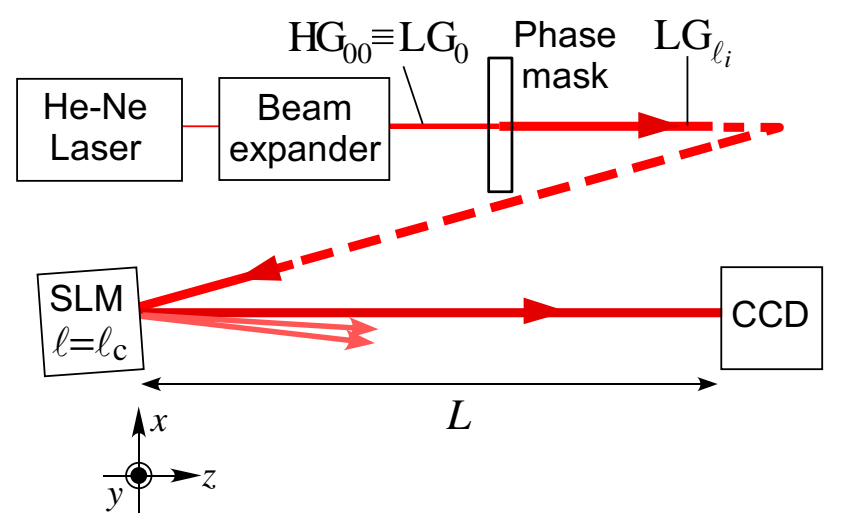

Figure 3: Experimental setup. Hermite-Gaussian $\left(\mathrm{HG} \equiv \mathrm{LG}_{\ell=0}\right)$ beam of He-Ne laser $(\lambda=633 \mathrm{~nm})$ is converted to a beam with $\ell=\ell_{i}$ by a phase mask. The OAM spectrum is evaluated by $\mathrm{LG}_{\ell} \rightarrow \mathrm{HG}$ mode conversion using a spatial light modulator (SLM). The cross-sectional profile of the 1st-diffraction beam is observed with a CCD camera.

\section{Experimental}

We now consider the experimental results of the $\mathrm{LG}_{\ell} \rightarrow \mathrm{HG}$ mode conversion. The setup is illustrated schematically in Fig. 3. To obtain a virtually pure LG mode from the He-Ne laser, we employed a phase mask (RPC Photonics, VPP-m633) consisting of the rotational phase patterns of $\ell_{i}=0$ to 


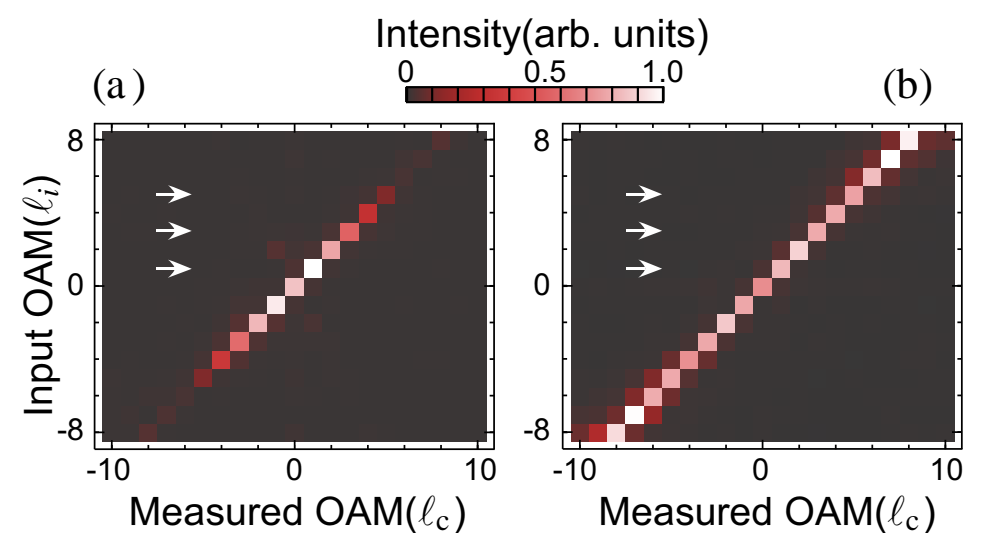

Figure 4: Density plots of the OAM spectra for the input LG beams with $\ell_{i}$ obtained by using (a) rotational phase modulations and (b) spiral phase modulations. Combination with a grating modulation is employed in both phase modulations. To compare the conversion efficiency, the intensity range in each plot is fixed at the highest maximum value. The arrows indicate the positions of $\ell_{i}(=1,3,5)$ for Fig. 5 .

8. The opposite signs of $\ell_{i}$ can be adopted by changing the side of the phase mask. The CGH on a liquid crystal SLM (Hamamatsu Photonics, X10468-07) was used to resolve the OAM. To spatially isolate the modulated component of the input beam, a stripe (grating) pattern was superimposed on the rotational or spiral phase modulation (typical CGH patterns are shown in the inset of Fig. ), and a 1st order diffraction beam was detected with an 8-bit CCD camera. The intensities of the converted HG were evaluated by selecting the same area of the CCD images for various OAM $\left(\ell_{c}\right)$. Since the sign of $\ell$ depends on the number of mirrors, we adjusted it so that the measured OAM $\ell_{c}$ for the spectral peak equaled $\ell_{i}$. With spiral phase modulation, different $f$ values were used to compensate the uniformity of the spectra.

The density plots of the OAM spectra are shown in Fig. 4 (a) and (b), in which the intensities of the spectra are normalized by the highest maximum 

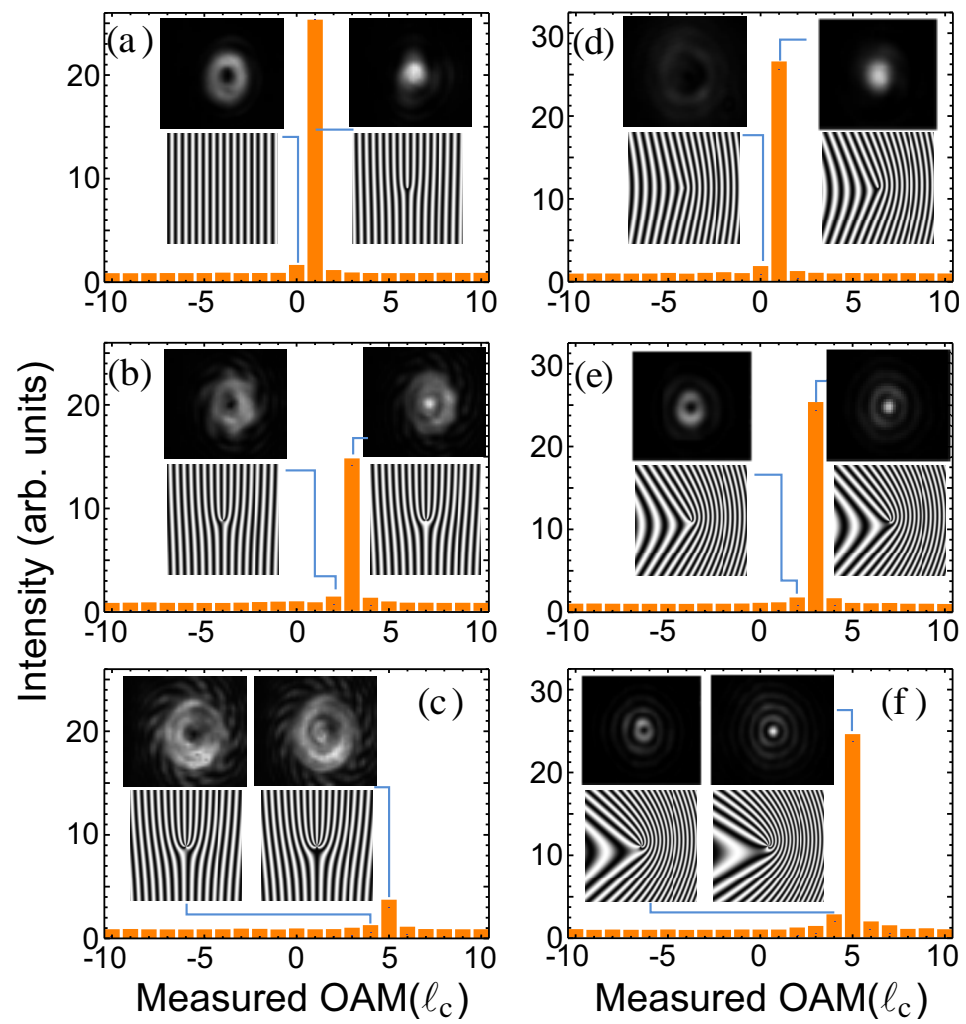

Figure 5: (a-c) The OAM spectra in Fig. 4 (a) and (d-f) in Fig. 4 (b) (cross-sectional data at the positions indicated by arrows in Fig. 4). The intensity range in each spectrum is fixed at the highest maximum value. The insets show the typical intensity profiles of the beams (above) diffracted by CGH patterns on SLM (below).

value in each plot so as to enhance the difference between the spectral uniformity of rotational and spiral phase modulations. Although both results show a single peak at $\ell_{c}=\ell_{i}$ in each analyzed $\mathrm{LG}_{\ell_{i}}\left(\ell_{i}=-8\right.$ to +8$)$, its intensity using rotational phase modulation decreases significantly with increasing $\left|\ell_{i}\right|$. On the other hand, the peak intensity with the spiral phase modulation is almost constant across various $\ell_{i}$. As suggested by the model calculation at the fixed propagation distance (bottom panels in Fig. 1), the HG beam 
converted by rotational phase modulation shows the ring-shaped component associated with the incomplete conversion, which decreases the intensity of the converted beam after the spatial filtering of the Gaussian-shaped component. In Fig. 4(a), the peak intensity distribution across $\ell_{c}=\ell_{i}$ is thus attributed to the variation of the conversion efficiency of the $\mathrm{LG}_{\ell} \rightarrow \mathrm{HG}$. The strong reduction of the peak at higher $\left|\ell_{i}\right|$ values convincingly follows the model calculation where the imperfect LHMC component becomes significant with increasing $\left|\ell_{i}\right|$. In other words, the uniform peak intensity obtained by the spiral phase modulation indicates that the efficiency of $\mathrm{LG}_{\ell} \rightarrow \mathrm{HG}$ is greatly improved in Fig. 4(b).

Figure 5 shows the OAM spectra for $\ell_{i}=1,3$, and 5 , whose positions are indicated by arrows in Fig. 4. The left $((\mathrm{a})-(\mathrm{c}))$ and right panels $((\mathrm{d})-(\mathrm{f}))$ show the results obtained with the rotational and spiral phase modulations, respectively. For $\ell_{c}=\ell_{i}$ and $\ell_{c}=\ell_{i}-1$, the CGH patterns are schematically illustrated in the lower part of the inset in each spectrum. The upper parts of the insets show the corresponding CCD images of the diffracted beam. As shown in the CCD images of Fig. 5(d)-(f), near-complete conversion to the HG is successfully realized using spiral phase modulation, which is in contrast to the results obtained with rotational phase modulation (Fig. $5(\mathrm{a})-(\mathrm{c}))$. Moreover, we again confirm the consistency with the model calculations; the CCD images for $\ell_{c}=\ell_{i}$ agree well with the results shown in the bottom panels of Figs. 1 and 2. Specifically, with rotational phase modulation, the ring-shaped imperfect component appears around the center peak for $\ell_{i}=3$ and 5 (see the insets in Fig. 5(b) and (c)), indicating that the imperfection of the intensity conversion remains and reduces the corre- 
sponding spectral intensity. It is also clear that the fraction of the imperfect component increases with $\left|\ell_{i}\right|$, which is connected to the OAM dependence of the conversion efficiency as mentioned above. It should be noted that the variation in the conversion efficiency affects not only the peak mode but also the side modes. The uniform conversion efficiency achieved by spiral phase modulation thus increases the precision of the OAM spectra.

\section{Summary}

Near-complete $\mathrm{LG}_{\ell} \rightarrow \mathrm{HG}$ mode conversion using spiral phase modulation was demonstrated for precise OAM spectroscopy. The model calculations indicated that the imperfection of the $\mathrm{LG}_{\ell} \rightarrow \mathrm{HG}$ using rotational phase modulation arises from the incomplete ring-shaped component of the converted HG owing to the diffraction process within the fixed propagation distance. Since the spiral phase focuses the diffraction beam within a finite propagation distance, the $\mathrm{LG}_{\ell} \rightarrow \mathrm{HG}$ using SPM produces a large overlap of its ring-shaped intensity. The cross-sectional views of the converted beams indicated that the mode selectivity using spiral phase modulation was greatly improved compared with that using the conventional rotational phase modulation. The OAM spectra for LG beams with various OAM also revealed the uniformity of the conversion efficiency.

\section{References}

[1] L. Allen, M. W. Beijersbergen, R. J. C. Spreeuw, J. P. Woerdman, Orbital angular-momentum of light and the transformation of laguerregaussian laser modes, Physical Review A 45 (1992) 8185-8189. 
[2] A. M. Yao, M. J. Padgett, Orbital angular momentum: origins, behavior and applications, Adv. Opt. Photon. 3 (2011) 161-204.

[3] T. Kuga, Y. Torii, N. Shiokawa, T. Hirano, Y. Shimizu, H. Sasada, Novel optical trap of atoms with a doughnut beam, Physical Review Letters 78 (1997) 4713-4716.

[4] M. Koyama, T. Hirose, M. Okida, K. Miyamoto, T. Omatsu, Power scaling of a picosecond vortex laser based on a stressed yb-doped fiber amplifier, Optics Express 19 (2011) 994-999.

[5] M. Berry, Making waves in physics - three wave singularities from the miraculous 1830s, Nature 403 (2000) 21-21.

[6] F. Flossmann, U. T. Schwarz, M. Maier, M. R. Dennis, Polarization singularities from unfolding an optical vortex through a birefringent crystal, Physical Review Letters 95 (2005).

[7] M. W. Beijersbergen, R. P. C. Coerwinkel, M. Kristensen, J. P. Woerdman, Helical-wavefront laser beams produced with a spiral phaseplate, Optics Communications 112 (1994) 321-327.

[8] V. Y. Bazhenov, M. V. Vasnetsov, M. S. Soskin, Laser beams with screw dislocations in their wavefronts, JETP Letters 52 (1990) 429-431.

[9] N. R. Heckenberg, R. McDuff, C. P. Smith, A. G. White, Generation of optical-phase singularities by computer-generated holograms, Optics Letters 17 (1992) 221-223. 
[10] A. Mair, A. Vaziri, G. Weihs, A. Zeilinger, Entanglement of the orbital angular momentum states of photons, Nature 412 (2001) 313-316.

[11] Y. Awaji, N. Wada, Y. Toda, Demonstration of spatial mode division multiplexing using laguerre-gaussian mode beam in telecom-wavelength, in: IEEE Photonics Society, 2010 23rd Annual Meeting of the, pp. 551552.

[12] R. Celechovsky, Z. Bouchal, Optical implementation of the vortex information channel, New Journal of Physics 9 (2007).

[13] L. Torner, J. P. Torres, S. Carrasco, Digital spiral imaging, Optics Express 13 (2005) 873-881.

[14] G. Gibson, J. Courtial, M. J. Padgett, M. Vasnetsov, V. Pas'ko, S. M. Barnett, S. Franke-Arnold, Free-space information transfer using light beams carrying orbital angular momentum, Optics Express 12 (2004) $5448-5456$.

[15] Y. Tokizane, K. Shimatake, Y. Toda, K. Oka, M. Tsubota, S. Tanda, R. Morita, Global evaluation of closed-loop electron dynamics in quasione-dimensional conductors using polarization vortices, Optics Express 17 (2009) 24198-24207.

[16] G. C. G. Berkhout, M. P. J. Lavery, J. Courtial, M. W. Beijersbergen, M. J. Padgett, Efficient sorting of orbital angular momentum states of light, Physical Review Letters 105 (2010). 